\title{
Contents
}

\section{Part I Overview}

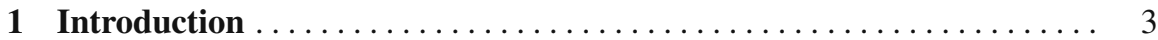

References ................................. 7

\section{Part II Further Essentials}

2 'Observational' Quantum Cosmology $\ldots \ldots \ldots \ldots \ldots \ldots \ldots \ldots \ldots \ldots$

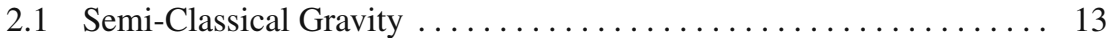

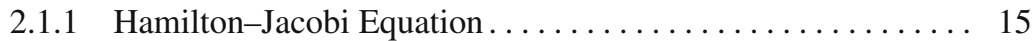

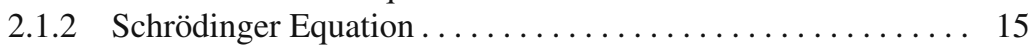

2.1.3 Quantum Gravity Corrections ................... 17

2.2 'Predictions' from Quantum Cosmology .................... 19

2.2.1 Towards Structure Formation $\ldots \ldots \ldots \ldots \ldots \ldots \ldots \ldots$

2.2.2 Towards Spacetime Decoherence ................ 21

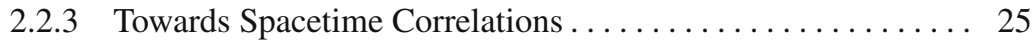

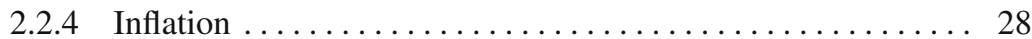

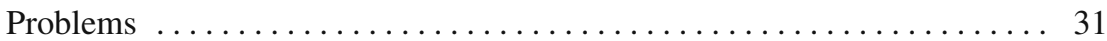

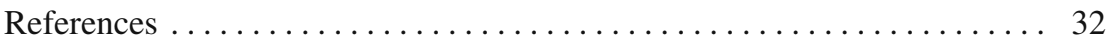

3 Additional SUSY and SUGRA Issues $\ldots \ldots \ldots \ldots \ldots \ldots \ldots \ldots \ldots$

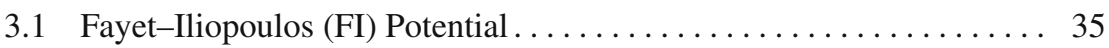

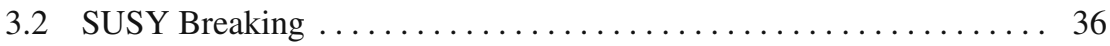

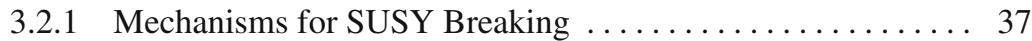

3.2.2 Spontaneous SUSY Breaking (in SUGRA) . . . . . . . . . 39

$3.3 N=2$ Supersymmetric Quantum Mechanics .............. 43

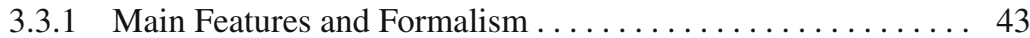

3.3.2 SQM, Topology, and Vacuum States ............. 52

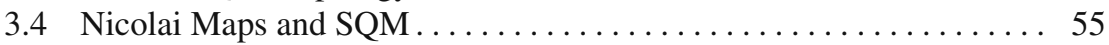

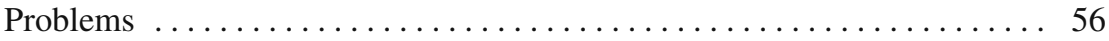

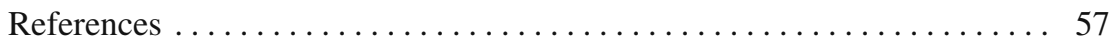




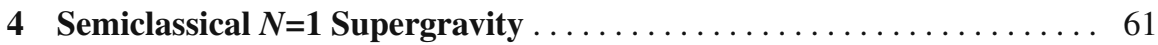

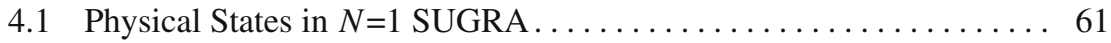

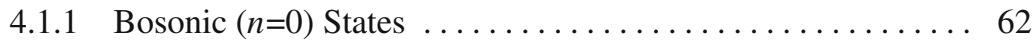

4.1.2 States with Finite Fermion Number ............... 63

4.1.3 Solutions with Infinite Fermion Number . . . . . . . . . . 63

4.2 Semiclassical Expansion ............................ 66

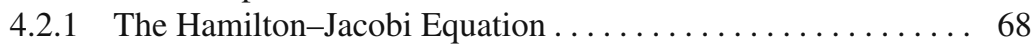

4.2.2 The DeWitt Supermetric . . . . . . . . . . . . . . . . . 71

4.2.3 The Schrödinger Equation .................... 72

4.2.4 The DeWitt Supermetric and the Schrödinger Equation..... . 74

4.2.5 Quantum $N=1$ SUGRA Corrections ................ 75

4.2.6 The DeWitt Supermetric and Quantum Gravity Corrections for the Schrödinger Equation .................. 78

4.2.7 Towards SQC with 'Observational' Insights? .......... 81

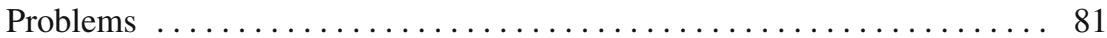

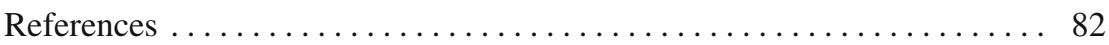

\section{Part III Complementary Frameworks in SQC}

5 Further Explorations in SQC $\boldsymbol{N}=1$ SUGRA $\ldots \ldots \ldots \ldots \ldots \ldots \ldots$

5.1 The Issue of Supersymmetry Breaking $\ldots \ldots \ldots \ldots \ldots \ldots \ldots \ldots . \ldots \ldots$

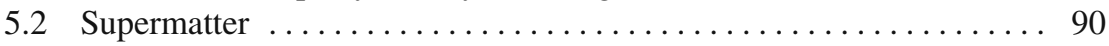

5.2.1 Generic Gauge Supermatter in FRW . . . . . . . . . . 91

5.2.2 Scalar Supermultiplets in Bianchi IX .............. 96

5.2.3 Vector Fields in Bianchi I $(N=2$ SUGRA $) \ldots \ldots \ldots \ldots 101$

Problems .......................................... 107

References ..................................... 108

6 Connections and Loops Within SQC $\ldots \ldots \ldots \ldots \ldots \ldots \ldots \ldots \ldots \ldots \ldots \ldots \ldots$

6.1 A Brief Summary of the Connection Representation............ 111

6.1.1 Two Spinor Representation . . . . . . . . . . . . . . . . . 111

6.1.2 Hamiltonian Formulation........................ 114

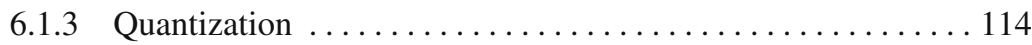

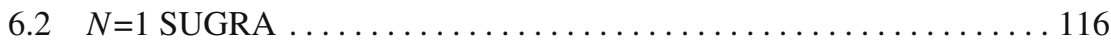

6.2.1 Hamiltonian Formulation..................... 116

6.2 .2 Quantization ................................ 117

6.2.3 FRW with Cosmological Constant ................. 118

6.2.4 Bianchi Class A Models .......................... 120

Problems ........................................ 123

References ..................................... 124

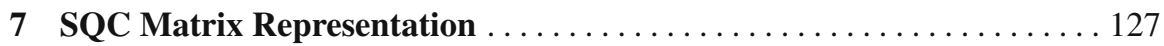

7.1 Motivation and Historical Background ................... 129 
7.2 Supersymmetric Bianchi Models . . . . . . . . . . . . . . . . . . 130

7.2.1 Bianchi I Cosmology . . . . . . . . . . . . . . . . . . . . . . . 133

7.2 .2 Bianchi IX Cosmology . . . . . . . . . . . . . . . . . . 138

7.2 .3 Taub Minisuperspace ........................... 143

7.2.4 FRW Minisuperspace ............................. 147

7.3 Bianchi Models and Lorentz Invariance . . . . . . . . . . . . . . . . . 147

7.3.1 Implications of the Lorentz Constraints . . . . . . . . . . . 148

7.3 .2 Physical States ................................. 153

7.3.3 Lorentz Constraint Components and Bianchi IX Diagonal Models .................................. 155

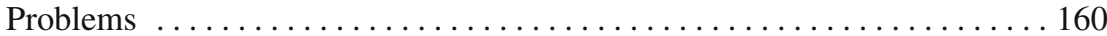

References ...................................... 160

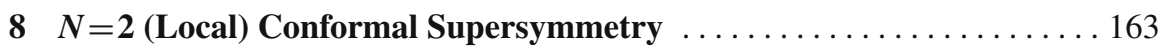

8.1 Motivation and Superfield Description $\ldots \ldots \ldots \ldots \ldots \ldots \ldots \ldots \ldots \ldots$

8.1.1 Empty Matter Sector ............................ 164

8.1 .2 Complex Scalar Fields ........................ 172

8.2 Quantum FRW Minisuperspace $\ldots \ldots \ldots \ldots \ldots \ldots \ldots \ldots \ldots \ldots \ldots$

$8.2 .1 \quad$ Supersymmetry Breaking....................... 183

8.2.2 Towards an Inflationary Scenario $\ldots \ldots \ldots \ldots \ldots \ldots \ldots \ldots$

Problems .......................................... 187

References .................................... 187

\section{Part IV More Points of Departure}

9 More Obstacles and Results: From QC to SQC ................ 191

9.1 Quantum Cosmology ................................. 191

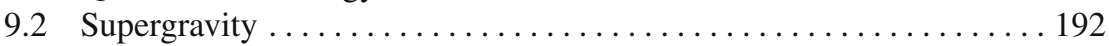

9.3 Supersymmetric Quantum Cosmology . . . . . . . . . . . . . . . . 193

9.3.1 FRW Models ................................... 193

9.3.2 Spatial Anisotropy ................................ 194

References ..................................... 195

10 More Routes Beyond the Borders . . . . . . . . . . . . . . . . . . . . . 197

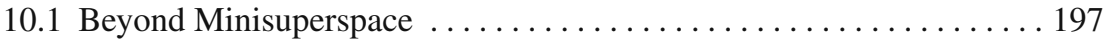

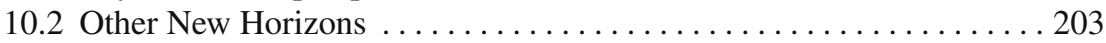

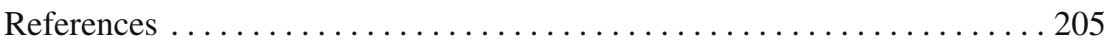

A List of Symbols, Notation, and Useful Expressions _.............. 207

A.1 List of Symbols .................................... 207

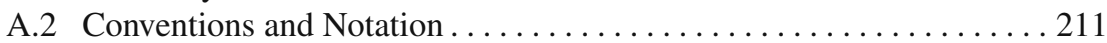

A.3 About Spinors .................................. 212

A.3.1 Spinor Representations of the Lorentz Group ............. 212 


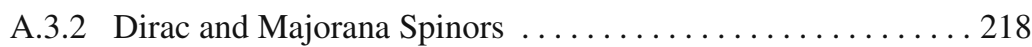

A.4 Useful Expressions . . . . . . . . . . . . . . . . . . . 222

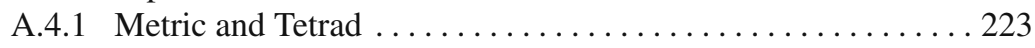

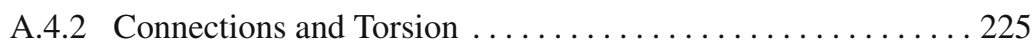

A.4.3 Covariant Derivatives ..................... 227

A.4.4 (Gravitational) Canonical Momenta . . . . . . . . . . . . 227

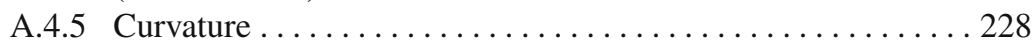

A.4.6 Decomposition with Four-Component Spinors . . . . . . . . . . 229

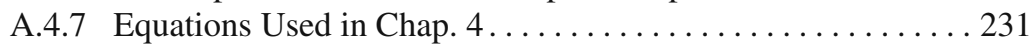

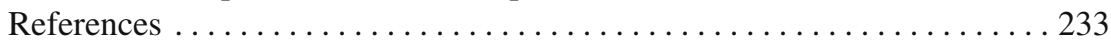

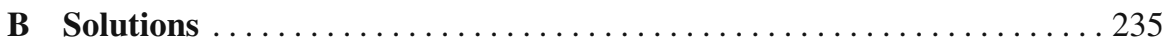

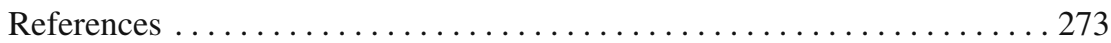

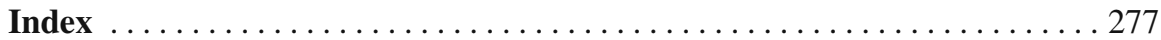

